\title{
Evaluation and Comparisons of Migration Techniques From IPv4 To IPv6 Using GNS3 Simulator
}

\author{
Ghaida Yagoub Ahmed Yosif Al-Gadi ${ }^{1}$, Dr. Amin Babiker A/Nabi Mustafa ${ }^{2}$, \\ Mahmoud Ahmed Hamied ${ }^{3}$ \\ ${ }^{1}$ Faculty Of Engineering, Neelain University ,Khartoum -Sudan \\ ${ }^{2}$ Faculty Of Engineering Neelain University, Khartoum-Sudan \\ ${ }^{3}$ Mysore University (India) SUDACAD -Sudan
}

\begin{abstract}
The enormous boom of the internet services and with the increased number of the devices, and applications that use the Internet, major changes in the overall architecture has required the use of new technologies. In this day became necessary to find a version of IPv6 (Internet Protocol Version 6) in order to reach out to the product meets all of these requirements and enable a global environment where processing rules clasped all applications. This paper discusses ways to migrate from IPv4 to IPv6,It uses GNS3 to simulate three ways used in transition to IP6 and Svolarwinds to analysis three parameters of network performance Latency, Packet loss. We tried to present in this paper a groundwork examination of IPv6 in Network migration and performance analysis, in which determine that network migration process is a very essential footstep in shaping how the communication will occur and will support with or detract from the enforcement of IPv6 policies[1].
\end{abstract}

Keywords: - IPv4, IPv6, GNS3, Tunneling,Dual Stack, NAT-PT

\section{INTRODUCTION}

The great expansion of the internet, these days, creates more significant challenges. Not only the addressing of new hosts like computer, tablets, laptop, cell phone but also the technologies. This requires an improvement in the overall architecture of the Internet to support the increase number of users, application, and services that use the Internet.

Internet Protocol version 6 (IPv6) came to meet the needs that enable us to get all the applications with high transparency and enable inside network environment rules. IPv6 makes important improvements to network topologies dynamically (exp. Peer to peer, client/server or mesh networks). Also, it improves most of networks functions like, notably in security, mobility, auto configuration, quality of service (QOS) and multicasting [2].

IPv6 is proposed to provide Internet with a larger address space and better performance [3]. In the past ten years, a lot of work has been done on the protocol design, connection and routing mechanism, and transition mechanism of IPv6. As the demand of IPv6-supported network equipment increases, some proposals are introduced to recommend methods for the evaluation of performance and platforms that focus on hardware and compatibility with IPv6 protocols [2].

After its first decade of protocol design and testing, the IPv6 Internet is now in a transition phase from experimental research networks of global operational networks.

Once a network begins to provide public services, its performance is always a big issue. As forIPv6, it is even more complex question mark.

\section{TRANSITION}

Moving from IPv4 to IPv6 will not need to be over all areas at the same time because such approach is difficult and it will take time. In addition, public networks now days has no single point of control so it is not possible to enforce like this change. In addition, such change to most companies and organizations already connected to the internet is unwelcome and unprofitable. The best approach for this move is to implementIPv6 along with existing IPv4.To make this approach much easier and coexist with existing IPv4 many transition techniques have been implemented [4]. There are three types: Dual Stack, Tunneling and Translation (NATPT).

A. Dual Stack

Dual Stack in simple way mean two protocols stack work in the same time or in parallel, the device will have both IPv4 and IPv6 protocol stacks [4]. For example, IPv4 will be used to communicate with IPv4 destinations, and IPv6 will be used to communicate with IPv6 destinations. 


\section{B. Tunneling}

When packets move between different places it will be encapsulated that is mean IPv6 packet inside IPv4packet, orIPv4 packet inside IPv6 packet [6].

\section{Translation}

Translation is a way to make a connection between networks that is used, differentprotocols;ittranslatesIPv6 traffic to IPv4 and IPv4 traffic to IPv6 when needed. One of translation methods is Network Address Translation-Protocol (NAT-PT) [5]. With this method of translation can be configured statically or dynamically to translate IPv6 address to IPv4 or IPv4 address to IPv6.

\section{METHOD}

We use GNS3 [7], software to simulate three different methods in the transition from IPv4 to IPv6 (Tunneling, Dual Stack, NAT-PT). In addition, using Solarwinds software for analysis the traffic between multiple destination, sand to measure the performance of the network at every one of the transition methods according to three parameters (Latency, Packet loss, response time).

\section{A. Simulation Environment Dual Stack}

The below Figure.1 illustrate the Dual Stack Connections.

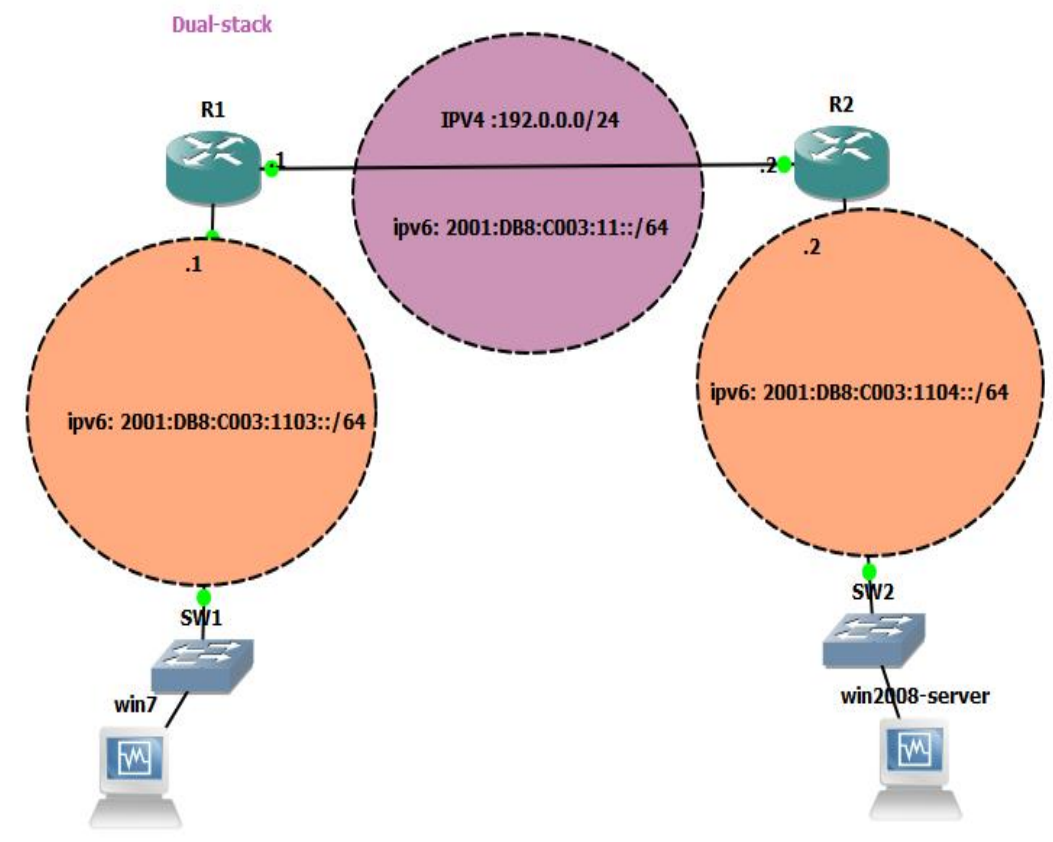

Figure 1: Dual-stack

In this simulation the components are:

- Two routers (using Cisco routers 3700). two switches-with Ethernet cabling (Fast Ethernet).

- Host windows server 2008 (with Solarwinds installation for analysis requirements) host windows 7 clients. (Using virtual box installation in both hosts for client and server)

- Addressing between router - PC addressing: IPv6

- Addressing protocol between router 1 and Router 2: Dual Stackboth (IPv4, IPv6). 


\section{B. Simulation Environment Tunneling}

This Figure. 2 show the Tunnel technique ofIPv6 to IPv6 network, while the transit network use IPV4.

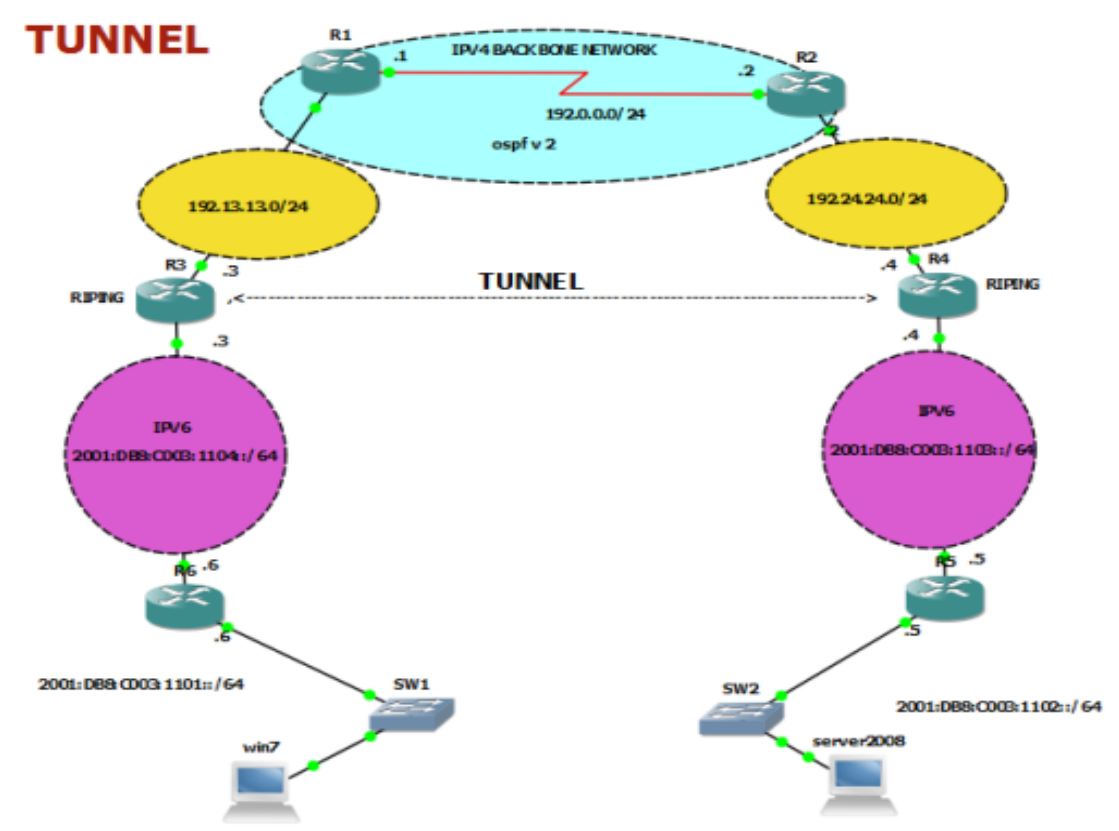

Figure 2: Tunnel

GNS3 topology implemented to test the tunneling can be seen in Figure (2) the test consists of six family Cisco routers (Cisco routers 3700) two computers. Two switches. Implemented through VirtualBox represented by the two computers.

Devices used:

- 6 Routers R1, R2 IPv4 backbone

1) Addressing protocol between R1, R3: IPV4 only

2) Addressing protocol between R2, R4: IPV4 only

3) Addressing protocol between R3, R6: IPV6 only

4) Addressing protocol between R4, R5: IPV6 only

Tunneling will take place from R3 to R4 (tunnel technique GRE).

\section{Simulation environment NAT-PT}

In this Figure. 3 shows the NAT-PT, IPV4 to IPV6 dynamic NAT translation.

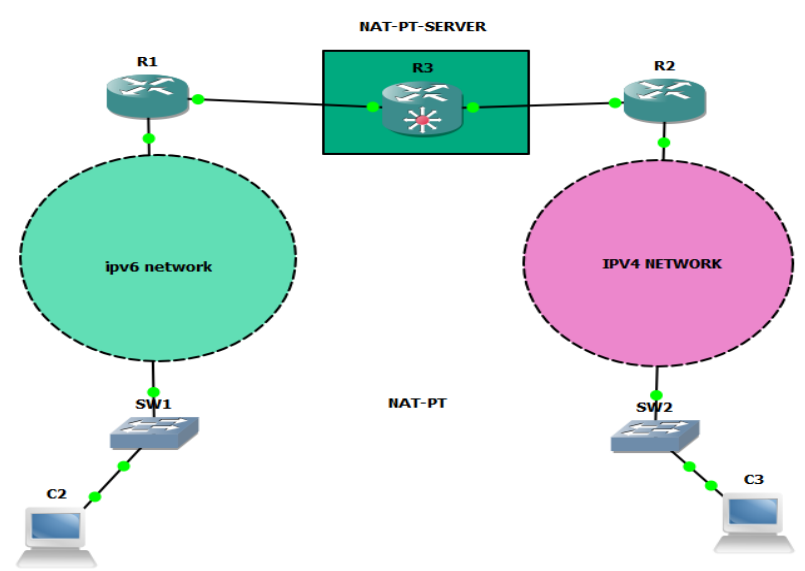

Figure 3: NAT-PT 
Components in the NAT-PT network.

- 1 host, windows server 2008 (with solarwind installation for analysis requirements)

- 2 switches for connecting.

2 hosts win 7 operating system as a client. (Using virtual box installation in both hosts for client and server)

Can be seen GNS3 topology NAT-PT translation configuration from IPv4 to IPv6 and from IPv6 to IPv4) implementation of the test in Figure 3, the test consists of a family:

- $\quad$ Two Router (using Cisco routers 3700)

- Two switches for connecting.

- $\quad$ Server (NAT-PT server) installed with solar winds) and connected to the computer running windows server. The first portion of the topology is using IPv4 (host wins $7+$ R3) the second part is using IPv6 (host c3 + $\mathrm{R} 2)$.

\section{A. Dual Stack network result}

\section{RESULT}

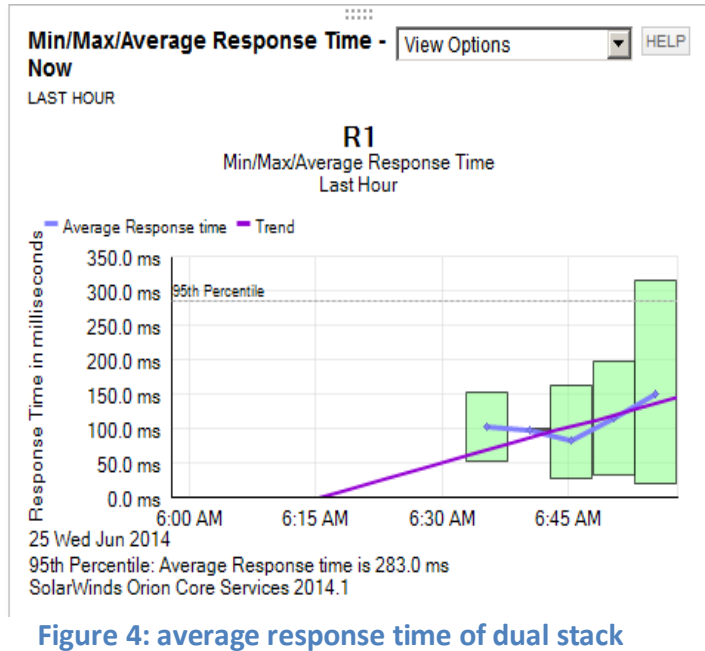

The average response time of dual stack $238 \mathrm{~ms}$ was measured in the above Figure.4 and the results show as packet size increase the response time increases.

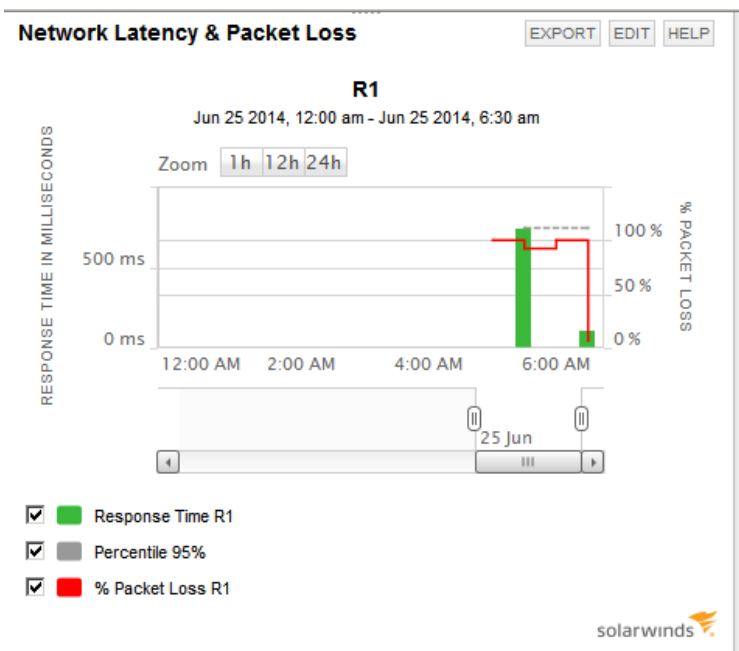

Figure 5: Network latency\& packet loss of Dual Stack

The average response time of Dual Stack high and packet loss high, show Figure.5. 
B. Tunnel network result

The average response time of Tunnel $=36 \mathrm{~ms}$ in the Previous Figure. 6 .

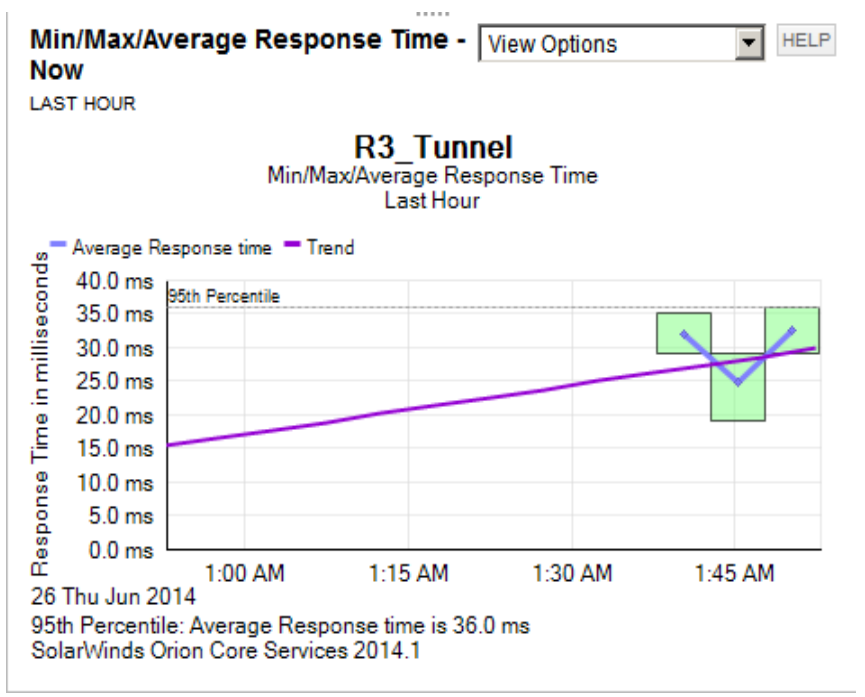

Figure 6: average response time of Tunnel

In Figure.7, there are fewlatency\& packet losses of the tunnel.

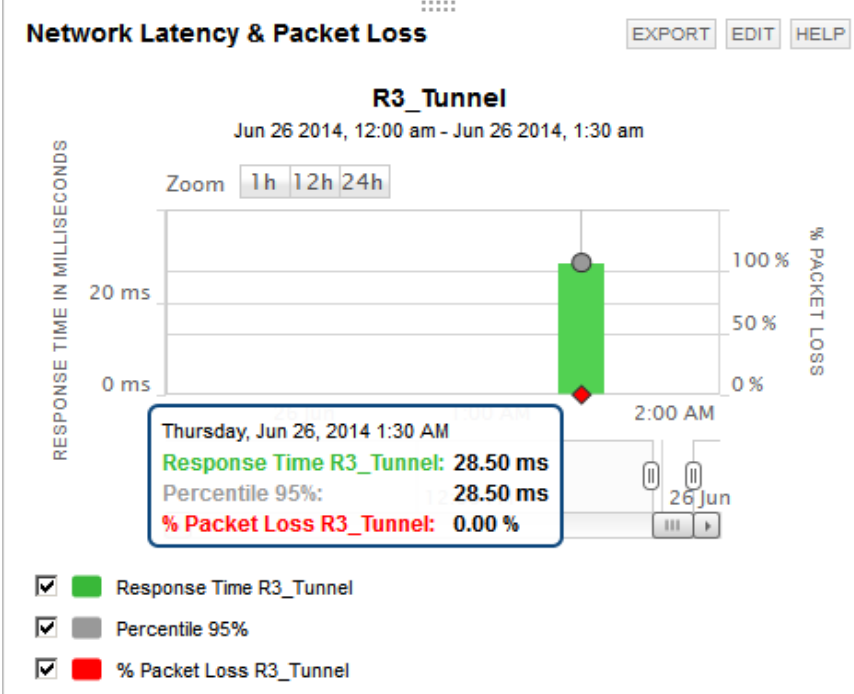

Figure 7: Networklatency\& packet loss of Tunnel

In the Figure. 6,7 we find that the average response time is equal to $28.5 \mathrm{~ms}$ and the loss of packets is equal to zero. 


\section{NAT-PT network result}

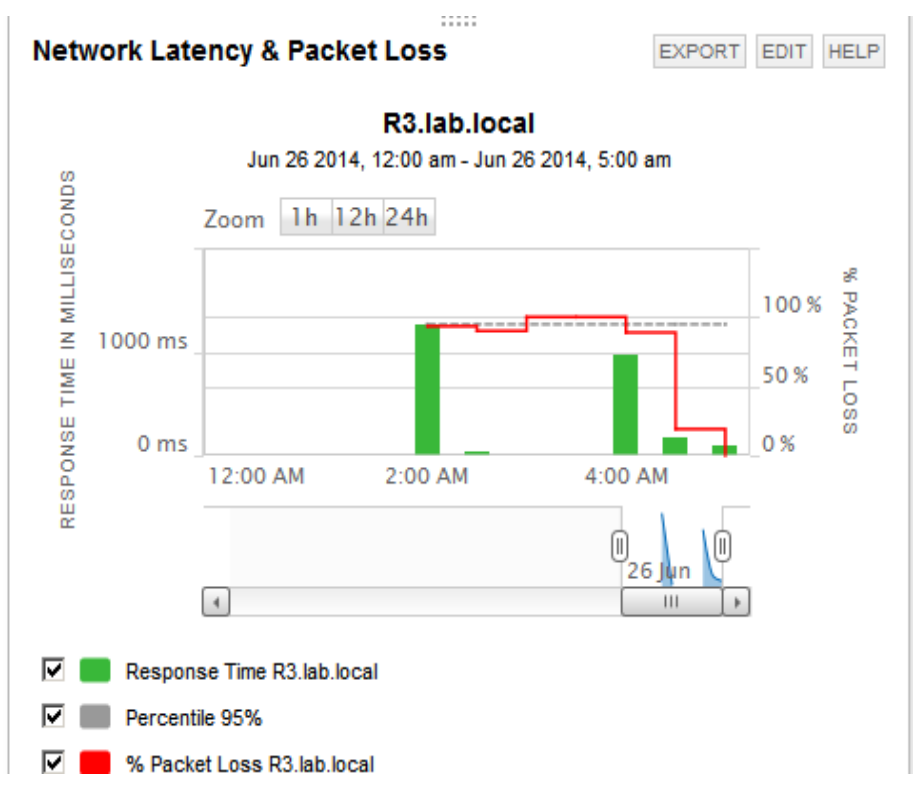

Figure 8: Networklatency\& packet loss of NAT-PT

In the above Figure.8, we measured network latency in ms.

The packet loss of NAT-PT was high; this is because of the above-mentioned facts The MTU and dual Translation.

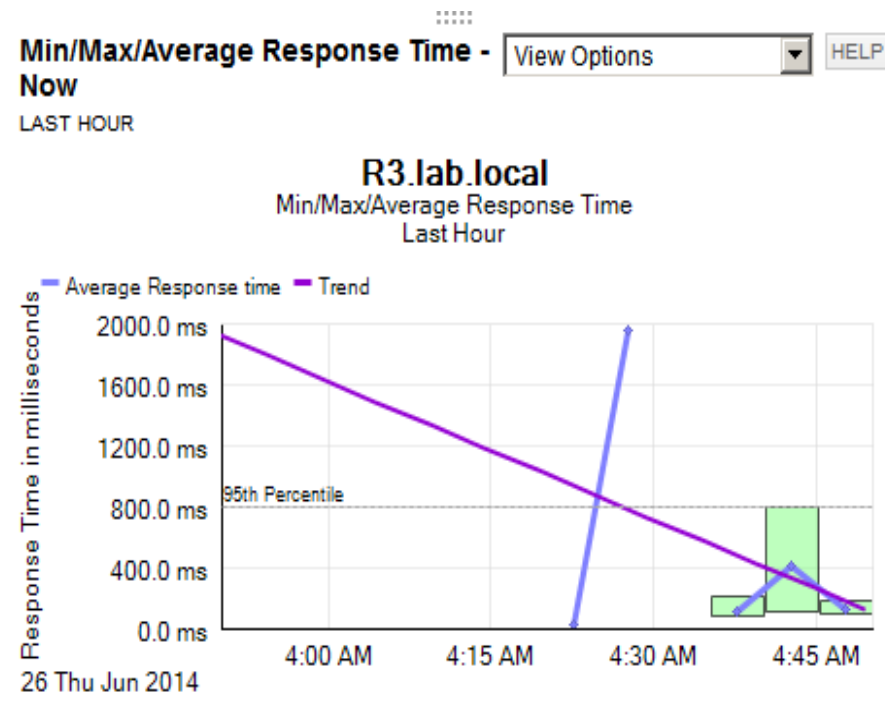

Figure 9: AVERAGE RESPONSE TIME NAT-PT

In Figure. 9 the average response time in of NAT-PT $=800 \mathrm{~ms}$ as measured in the above figure10. Moreover, the result shows as packet size increases the response time increases.

We absorb high packet loss and latency of the three proposed migration techniques in NAT-PT, these due to that the fact dual (two-way) translation technique and tunneling.

\section{CONCLUSIONS}


The migration from IPv4 to IPv6 is a something of an evident in the long term, however, IPv6 does not only provides IP address space - there are some other advantages including long-term cost savings and better performance. In addition to the realization of true interoperability and mobility of networks. In this paper, we conducted three scenarios, models of transition technique. Tunnel, NAT-PT and Dual Stack. We have implemented the experiment by using GNS 3 and analyze the work of the three networks through the Solarwinds program .And we measure packet loss and latency and Response Time.

We have found that the average response time is different from one network to the other, and that the adoption of the topology used by each network and the number of machines in it. We find that the average response time in the tunnel is equal to $28.5 \mathrm{~ms}$ and in the Dual Stack is $283 \mathrm{~ms}$ As far as NAT-PT an average response time of $800 \mathrm{~ms}$. The higher average response time resulted in higher latency. There is higher latency in NAT-PT fellows Dual Stack and last tunnel. We conclude that from the simulation of these three techniques, that the tunneling technique is the best in terms of latency, packet loss, and RRT.

\section{REFERENCE}

[1] MD. tarig Aziz, Mohamed Saiful Islam, MD. nazmul Islam khan. Throughput performance evolution of video /voice traffic in IPv4/IPv6 network. International journal of computer applications. December 2, 2011, p, 1.

[2] A.Dutta, J.ALberi, A.Cheng, B.Horgan, T.McAuley, D.Chee, B.Lyles, "IPv6TRANSITIONTECHNIQUSFORLEGACY APPLICATION":582, MILCOM 2006.

[3] Ali, Amer nizar abu. Comparison study between IPv4\&IPv6. International journal of computer science issue. May 1, 2013.

[4] Internet Engineering Task Force (IETF) RFC6052, 3513, 4291, 6104, HTTP://tools.ietf. Or/htm/

[5] P. Loshin, IPv6: Theory, Protocol and Practice, Second Edition, 500 Sansome Street, Suite 400, San Francisco, CA 94111: Morgan Kaufmann Publishers, 2004.

[6] GNS3. "Hardware that GNS3 can emulate", http://www.gns3.org/hardware/.

[7] C. Bouras, P. Guanos, and A. Karaliotas, "The Deployment Of IPv6 in an IPv4 world and Transition strategies," Internet Research: Electronic Networking Applications and Policy, Vol. 13, no. 2, pp. 86-93, 2003. 